\title{
Stability and Robustness of L1-Adaptive Control of Time-delay System
}

\author{
Feng Yiwei ${ }^{1, a}$, Feng Ping ${ }^{2, b}$ \\ ${ }^{1}$ College of Electrical and Information Engineering, LanZhou University of Technolog \\ y, LanZhou, 730050, China \\ ${ }^{2}$ College of Electrical and Information Engineering, LanZhou University of Technolog \\ y, LanZhou, 730050, China \\ aemail: ywfeng@yeah.net, bemail: 540641470@qq.com
}

Keywords: Stability; Robustness; $L_{1}$-Adaptive Control; Delay

\begin{abstract}
The objective of this paper is the implementation and validation of an adaptive controller for linear systems with state delay. The contribution of this paper is the design of an adaptive controller that guarantees the stability and performance of the given system, even when the nominal conditions change. Some preliminary results are presented. The proposed approach is validated on a special model. Moreover, if we reduce the filter, compared to standard Model Reference Adaptive Control (MRAC), the performance of system can be deteriorated, even if without the state delay.
\end{abstract}

\section{Introduction}

The Model reference adaptive control (MRAC) is unquestionably the most widely studied problem and has a very long history going back to the 1950s and extending to the present time [1]. In the past decades, there has been increasing interest in the study of performance bounds of adaptive controllers for linear system in the presence of unmodeled dynamics and time-varying delays. The earliest at tempts to solve the MRAC problem followed the classical path of designing an observer, that had to be made adaptive because of the unknown plant parameters and then feeding back the observed state. Among these efforts, a research approach emerged that involves the insertion of a stable strictly proper filter, usually first order, at the input of a standard MRAC scheme. In such applications, the key problem is to know the performance bounds of the adaptive controllers. During its design and hardware implementation, however, the stability and performance of the system may be destroyed by its unavoidable uncertainty due to the existence of modeling errors, the delays in the state. As part of the approach, it is recommended to use very high adaptive gains for fast and robust adaptation. As a result, it is important to investigate the $\mathrm{L}_{1}$-adaptive robust controller of MRAC systems.

This paper is aimed at four points. The first point is, the insertion of the filter deteriorates. The second point that is due to the phase lag introduced by the input filter in the $\mathrm{L}_{1}$-AC, the stability margin of the system may be deteriorated. This, in turn, imposes a bound on the allowable parametric uncertainty. The third and final point is that the design recommendation of using very high adaptive gains,bring up two problems which have not been addressed in the present literature of $\mathrm{L}_{1}-\mathrm{AC}$. The first problem is that the use of high adaptive gains has a negative effect on robustness in the literature [8]-[11]. The second is that very high adaptive gains make the differential equation of the adaptive law stiff and difficult to solve numerically.

The rest of this paper is organized as follows: in Section 2 , the considered $\mathrm{L}_{1}$-AC problem is formulated and some definitions and lemmas are presented. Based on the given lemmas, the stability of the discussed system is derived in Section 3, meanwhile, a numerical example is showed to demonstrate the robustness of our results. Finally, conclusions are drawn in Section 4.

\section{Problem Fromulation}

In this section, we consider the following dynamic systems :

$$
\dot{x}(t)=A_{m} x(t)+b \theta^{* T} x(t-\tau)+b u(t)
$$




$$
y(t)=c^{T} x(t)
$$

where $x(t) \in R^{n}$ and $x(t-\tau) \in R^{n}$ are the current state and previous state vector and measurable, respectively, $\tau$ is the system delay and it is a bounded nonnegative constant , $x(0)=x_{0}, A_{m}$ is a known matrix and $\left(A_{m}, b\right)$ is controllable, $b, c$ are known constant vectors, $\theta^{*} \in R^{n}$ is an unknown parameter vector. According to the reference [6], the L1-AC design proposed in [6] to meet the control objective: design an adaptive controller to ensure that the system output $y(t)$ follows a give reference signal $r(t)$ with quantifiable transient and steady-state performance bounds.

$$
\begin{array}{lrl}
\dot{\hat{x}}(t) & =A_{m} \hat{x}(t)+b \theta^{T}(t) x(t-\tau)+b u(t), & \hat{x}(0)=x_{0} \\
\dot{\theta}(t)=\Gamma x(t-\tau) \tilde{x}(t)^{T} P b, & \theta(0)=\theta_{0}
\end{array}
$$

where $\tilde{x}(t)=x(t)-\hat{x}(t), P=P^{T}>0$ is the solution of the Lyapunov equation $A_{m}^{T} P+P A_{m}=-Q, Q$ is a positive definite matrix and $Q=Q^{T} \cdot \Gamma>0$ is the adaptive gain assumed to be a scalar as in [4]. Equation (4) is a standard parameter estimator scheme that described in [7].

Using a simple Lyapunov function:

$V=\tilde{x}^{T}(t) P \tilde{x}(t)+\tilde{\theta}^{T}(t) \Gamma^{-1} \tilde{\theta}(t)$

where $\tilde{\theta}(t)=\theta(t)-\theta^{*}$, we assumed that $V_{2}=\tilde{\theta}^{T}(t) \Gamma^{-1} \tilde{\theta}(t)$ and $V_{1}=\tilde{x}^{T}(t) P \tilde{x}(t)$,from $\tilde{x}(t)=x(t)-\hat{x}(t)$, $\dot{\tilde{x}}(t)=\dot{x}(t)-\dot{\hat{x}}(t)$, (1) and (3), there has a result that $\dot{\tilde{x}}(t)=A_{m} \tilde{x}(t)-b \tilde{\theta}^{T}(t) x(t-\tau)$ and we can get

$\dot{V}_{1}=\dot{\tilde{x}}^{T}(t) P \tilde{x}(t)+\tilde{x}^{T}(t) P \dot{\tilde{x}}(t)=\left(A_{m} \tilde{x}(t)-b \tilde{\theta}^{T}(t) x(t-\tau)\right)^{T} P \tilde{x}(t)+\tilde{x}^{T}(t) P\left(A_{m} \tilde{x}(t)-b \tilde{\theta}^{T}(t) x(t-\tau)\right)$

$=-\tilde{x}^{T}(t) Q \tilde{x}(t)-x^{T}(t-\tau) \tilde{\theta}(t) b^{T} P \tilde{x}(t)-\tilde{x}^{T}(t) P b \tilde{\theta}^{T}(t) x(t-\tau)$

$\dot{V}_{2}=\dot{\tilde{\theta}}^{T}(t) \Gamma^{-1} \tilde{\theta}(t)+\tilde{\theta}^{T}(t) \Gamma^{-1} \dot{\tilde{\theta}}(t)=\mathrm{b}^{\mathrm{T}} \mathrm{P}^{\mathrm{T}} \tilde{\mathrm{x}}(\mathrm{t}-\tau) \mathrm{x}^{\mathrm{T}}(\mathrm{t}-\tau) \tilde{\theta}(\mathrm{t})+\tilde{\theta}^{\mathrm{T}}(\mathrm{t}) \mathrm{x}(\mathrm{t}-\tau) \tilde{\mathrm{x}}^{\mathrm{T}}(\mathrm{t}-\tau) \mathrm{Pb}$

$\dot{V}=\dot{V}_{1}+\dot{V}_{2}=-\tilde{x}^{T}(t) Q \tilde{x}(t) \leq 0$, it means that $\tilde{\theta}(t), \tilde{x}(t)$ are bounded. Then, the control law which proposed by $\mathrm{L}_{1}$-AC can be obtained [5]-[7]

$u(t)=C(s)\left[-\theta^{T}(t) x(t-\tau)+k_{0} r(t)\right]$

where $C(s)$ is a stable, strictly proper, transfer function with $C(0)=1$ and $k_{0}=-1 /\left(c^{T} A_{m}^{-1} b\right)$. As point out in one of these studies [1], where $C(s)=1$ is simply the MRAC law

$$
u(t)=-\theta^{T}(t) x(t-\tau)+k_{0} r(t)
$$

The MRAC law (9) together with (3) guarantee that $x$ follows the state $\hat{x}$ of the reference model

$$
\dot{\hat{x}}(t-\tau)=A_{m} \hat{x}(t-\tau)+b_{m} r(t), b_{m}=k_{0} b
$$

Obviously, there are the following differences between $\mathrm{L}_{1}$-AC and MRAC. a) control objective: the MRAC objective is for the plant state/output to track the state/output of the reference model for any reference input signal $r(t)$, whereas the control objective of $\mathrm{L}_{1}$-AC is for the plant output to track the reference input $r(t)$; b) design schemes: the two schemes is just the insertion of a strictly proper stable filter $C(s)$ at the input of the MRAC. The important question in [7] also can be applied to the situation of delay, because of the result irrelevant to the delay. The closed-loop transfer function obtained by substituting (8) with $\theta(t)=\theta^{*}$ into (1) is given by

$$
y(t)=c^{T}\left(s I-A_{m}+b(C(s)-1) \theta^{* T} e^{-\tau s}\right)^{-1} b C(s)\left[k_{0} r(t)\right]
$$

From the small-gain theorem, we can get: if

$$
\left\|\left(s I-A_{m}\right)^{-1} b(C(s)-1)\right\|_{1}\left\|\theta^{*} e^{-\tau s}\right\|_{1}<1
$$

the closed-loop system is stable.

According to the certainty equivalence principle for the control objective of $y(t)=\lim _{t \rightarrow \infty} r(t)$ for any given bounded $r$, we need to show the existence of a $\theta^{*}$ that meet (12),and must guarantee that

$$
c^{T}\left(s I-A_{m}+b(C(s)-1) \theta^{* T} e^{-\tau s}\right)^{-1} b C(s) k_{0}=1, \forall s
$$

We can find that there is no $\theta^{*}$ can satisfy the equation (13) only if make the $s=0$ that because the left of (8) is a strictly proper transfer function, where $r$ is a constant because of $k_{0}=-1 / c^{T} A_{m}^{-1} b$ and $C(0)=1$. 
Lemma 1 in document [7] indicates that using the MRAC structure to track the reference input $r(t)$ at all kinds of frequencies is impossible for the only simple reason that the control objective of MRAC is to tracking the output of the reference model rather than its input, but the objective of the reference signal can be got by using other adaptive control schemes [10]. Why the scheme can track the constant reference input $r(t)$ ? That only because it can guarantee $y(t)=r(t)$ at the steady state where $C(0)=1$ and $k_{0}=-1 / c^{T} A_{m}^{-1} b$. Inequation (12) shows that the limitation of parametric $\theta^{*}$ in order to guarantee stability precisely. Lemma 1 clearly shows that both MRAC and $\mathrm{L}_{1}$-AC cannot be used to track the reference signal.

\section{Main Results}

Why the MRAC control objective was changed to track the reference input that cannot be met by choosing control structure, the reasons were not be identified in [1]-[3]. However, the Theorem which discussed in [1] shows that we can change the control objective, in other words, changing track the reference input to reference model output. For the Theorem in [1], we have the closed-loop reference system:

$$
\begin{aligned}
& \dot{x}_{r e f}(t)=A_{m} x_{r e f}(t)+b \theta^{* T} x_{r e f}(t-\tau)+b u_{r e f}(t), \quad y_{r e f}=c^{T} x_{r e f}(t) \\
& u_{r e f}(t)=C(s)\left[-\theta^{* T} x_{r e f}(t-\tau)+k_{0} r(t)\right]
\end{aligned}
$$

where $x_{\text {ref }}(0)=x(0)$, obtained by replacing $\theta(t)$ with $\theta^{*}$ in (1)-(8). If condition (12) is satisfied, along with the close-loop system (1)-(8), they lead to the following results:

$$
\lim _{t \rightarrow \infty}\left\|x(t)-x_{\text {ref }}(t)\right\|=0, \lim _{t \rightarrow \infty}\left\|x(t-\tau)-x_{\text {ref }}(t-\tau)\right\|=0, \lim _{t \rightarrow \infty}\left|u(t)-u_{\text {ref }}(t)\right|=0
$$

and

$$
\left\|x(t)-x_{\text {ref }}(t)\right\|_{\infty} \leq \gamma_{0} / \sqrt{\Gamma},\left\|x(t-\tau)-x_{\text {ref }}(t-\tau)\right\|_{\infty} \leq \gamma_{1} / \sqrt{\Gamma},\left\|u(t)-u_{\text {ref }}(t)\right\|_{\infty} \leq \gamma_{2} / \sqrt{\Gamma}
$$

where $\gamma_{0}, \gamma_{1}$ and $\gamma_{2}$ are positive constants independent of $\Gamma$, and $\theta^{*}$ is unknown, the desired properties of the plant cannot be designed to represent with (14) and (15), from the system in [1], we have the following system as a desired reference model:

$$
\dot{x}_{\text {des }}(t)=A_{m} x_{\text {des }}(t)+k_{0} b r_{0}, \quad y_{\text {des }}(t)=c^{T} x_{\text {des }}(t), r_{0}=C(s)[r(t)]
$$

There is a lemma in [1] which can be used to compare the fictitious reference system in (13)-(14) with the desired reference model in (18) as

$$
\left\|y_{\text {def }}(t)-y_{\text {des }}(t)\right\|_{\infty} \leq \lambda /(1-\lambda)\|c\|_{1}\left\|k_{0} W_{b}(s) C(s)\right\|_{1}\|r(t)\|_{\infty}
$$

where $W_{b}(s)=\left(s I-A_{m}\right)^{-1} b, \lambda=\left\|W_{b}(s)(C(s)-1) e^{-\tau s}\right\|_{1} \theta_{\max }$, and $\theta_{\max }$ is an upper bound for the norm of $\theta^{*}$. From (19), we can see that minimizing the bound of $C(s)$ is $C(s)=1$. Put another way, this situation implies removing the strictly proper stable filter and using the standard MRAC scheme. The MRAC law (4), (9) and (10) as reference model which same as (11) where $C(s)=1$, guarantee a exact result. The following Theorem 1 is the conclusion.

Theorem 1: (i) The MRAC scheme (4), (9), (10) guarantee that all signals are uniformly bounded and tracking error $e(t)=x(t)-x_{m}(t)$ converges to 0 for all bounded reference inputs $r(t)$ where $x_{m}(t)=\hat{x}(t)$ is the state of the reference model (10). In addition, the tracking error satisfies the bound

$$
\|e(t)\|_{\infty} \leq v_{0} / \sqrt{\Gamma} \text {, if } e(0)=0
$$

where $v_{0}=\|\tilde{\theta}(0)\|_{2} / \sqrt{\lambda_{\min }\{P\}}$ is a constant independent of $\Gamma$. In addition, if $r(t)$ is large enough, $\theta(t) \rightarrow \theta^{*}$ and $x(t) \rightarrow x_{m}(t)$ are exponentially fast convergence, respectively.

(ii) The L1-AC scheme (3), (4), (8) guarantee the following: Restrict $\theta^{*}$ to satisfied (12). Suppose that the $C(s)=1 / \eta s+1$ is the form of strictly proper stable filter, where $\eta>0$ is a design parameter to be suitably chosen. There exists a $\eta_{\max }>0$ so that for any $\eta \in\left[0, \eta_{\max }\right)$ all signals are uniformly bounded and the tracking error satisfies 


$$
\|e(t)\|_{\infty} \leq \eta v_{1}+v_{2} / \sqrt{\Gamma} \text {,if } e(0)=0
$$

where $v_{1}=\left(\left\|s W_{b}(s)\right\|_{1}\left(\theta_{\max } \bar{X}_{m}+\bar{r}\right)\right) /\left(1-\eta / \eta_{\max }\right)$ and $v_{2}=v_{0} /\left(1-\eta / \eta_{\max }\right)$ are constants independent of $\Gamma$, $W_{\mathrm{b}}(s)=\left(s I-A_{m}\right)^{-1} b, \eta_{\max }=1 /\left(\theta_{\max }\left\|s W_{b}(s)\right\|_{1}\right.$, and the upper bounds of $\theta(t), x_{m}(t)$ and $k_{0} r(t)$ are $\theta_{\max }, \bar{X}_{m}$, and $\bar{r}$, respectively.

Proof: Using the Lyapunov candidate $V=e^{T}(t) P e(t)+\tilde{\theta}^{T}(t) \Gamma^{-1} \tilde{\theta}(t)$, where $P$ is the solution of $A_{m}^{T} P+P A_{m}=-I$, is imply all signals are uniformly bounded and that $e(t) \rightarrow 0$ as $t \rightarrow 0$, by first showing that $\dot{V}=-e^{T}(t) e(t) \leq 0$. It follows that

$$
\begin{aligned}
& e^{T}(t) P e(t) \leq V(t) \leq V(0)=\tilde{\theta}^{T}(0) \tilde{\theta}(0) / \Gamma, \forall t \text { if } e(0)=0 . \text { Then, we have } \\
& \lambda_{\min }\{P\}\left\|e_{t}\right\|_{\infty}^{2} \leq \lambda_{\min }\{P\}\left\|e_{t}\right\|_{2}^{2} \leq V(t) \leq \tilde{\theta}^{T}(0) \tilde{\theta}(0) / \Gamma
\end{aligned}
$$

where $\lambda_{\min }\{P\}>0$, so the (i) can be proved.

Similarly, it can be shown that $\|\tilde{x}(t)\|_{\infty} \leq v_{0} / \sqrt{\Gamma}$. Equation (3) can be written as

$$
\hat{x}(t)=W_{b}(1-C)\left[\theta^{T} \hat{x}(t-\tau)\right]+W_{b}(1-C)\left[\theta^{T} \tilde{x}(t-\tau)\right]+W_{b} C\left[k_{0} r(t)\right]+w(t)
$$

where $W_{b}(s)=\left(s I-A_{m}\right)^{-1} b$ and $w(t)=\ell^{-1}\left\{\left(s I-A_{m}\right)^{-1} x_{0}\right\}$

is a bounded decaying function. Then,

$$
\|\hat{x}(t)\|_{\infty} \leq\left\|W_{b}(1-C)\right\|_{1}\left\|\theta^{T} \tilde{x}(t-\tau)\right\|_{\infty}+\left\|W_{b}(1-C)\right\|_{1}\left\|\theta^{T} \hat{x}(t-\tau)\right\|_{\infty}+\left\|W_{b} C\right\|_{1}\left\|k_{0} r(t)\right\|_{\infty}+\|w(t)\|_{\infty}
$$

Then, with filter $C(s)=1 /(\eta s+1)$ we have

$$
\begin{gathered}
\|\hat{x}(t)\|_{\infty} \leq \eta \theta_{\max }\left\|s W_{b}\right\|_{1}\|1 /(\eta s+1)\|\|\hat{x}(t)\|_{\infty}+\eta v \theta_{\max } / \sqrt{\Gamma}\left\|s W_{b}\right\|_{1}\|1 /(\eta s+1)\|_{1}+v\left\|W_{b}\right\|_{1}\|1 /(\eta s+1)\|_{1}+v \\
=\eta \theta_{\max }\left\|s W_{b}\right\|_{1}\|\hat{x}(t)\|_{\infty}+\eta \frac{v \theta_{\max }}{\sqrt{\Gamma}}\left\|s W_{b}\right\|_{1}+v\left\|W_{b}\right\|_{1}+v \\
e(t)=W_{b}(1-C)\left[\theta^{T} e(t-\tau)\right]+W_{b}(1-C)\left[\theta^{T} x_{m}(t-\tau)-k_{0} r(t)\right]+\tilde{x}(t-\tau)
\end{gathered}
$$

Then, we obtain

$$
\|e(t)\|_{\infty} \leq \eta \theta_{\max }\left\|s W_{b}\right\|_{1}\|e(t)\|_{\infty}+\eta\left\|s W_{b}\right\|_{1}\left\|\theta^{T} x_{m}(t-\tau)-k_{0} r(t)\right\|_{\infty}+\|\tilde{x}(t)\|_{\infty}
$$

Since $\|\tilde{x}(t)\|_{\infty} \leq v_{0} / \sqrt{\Gamma}$, then $\forall t \in\left[0, \eta_{\max }\right)$ we have

$$
\|e(t)\|_{\infty} \leq \eta v_{1}+v_{2} / \sqrt{\Gamma}, \forall t \geq 0
$$

where $v_{1}, v_{2}>0$ are independent of $\Gamma$. The (ii) can be proved.

We can see that the standard MRAC satisfies its control objective from the Theorem 1 . It gets its control objective without the limitations on $\theta^{*}$ imposed in $\mathrm{L}_{1}$-AC indicated by (12). On the contrary, with non-constant reference inputs, the $\mathrm{L}_{1}$-AC scheme cannot meet the objective which guarantees a zero steady-state tracking error, furthermore, the tracking error of the computed transient bound is larger than that with MRAC. Given that in such a case, the performance of the MRAC is the same as that of $\mathrm{L}_{1}-\mathrm{AC}$, it is clear that the additional complexity of a filter offers no benefit and given that the standard MRAC can meet the control objective exactly and with better bounds.

Next, we discuss the robustness of $\mathrm{L}_{1}$-AC. The effect of unmodeled dynamics can be analyzed by inserting a multiplicative uncertainty term $\Delta_{m}(s)$ in the plant dynamics (1) as

$$
\dot{x}(t)=A_{m} x(t)+b \theta^{* T} x(t-\tau)+b\left(1+\Delta_{m}(s)\right)[u(t)]
$$

$\Delta_{m}(s)$, multiplicative uncertainty, may represent input delay and other modeling errors of unmodeled (actuator) dynamics [9]. We assumed that its structure and parameters are unknown. The question is whether the presence of a nonzero unknown $\Delta_{m}(s)$ can be tolerated when a control scheme designed for $\Delta_{m}(s)=0$, and if so, what is the size of the allowable uncertainty, asked in robust adaptive control [9], [10] as well as in robust control [12]. The question is whether the use of the input filter in (8) can improve robustness with respect to the unknown $\Delta_{m}(s)$. As mentioned before, let us examine what stability properties can be obtained when we have perfect parameter information with the certainty equivalence principle, i.e., $\theta(t)=\theta^{*}$ in the control law. The control law in such a case is given by

$$
u(t)=C(s)\left[-\theta^{* T} x(t-\tau)+k_{0} r(t)\right.
$$

and $C(s)=1 / \eta s+1, \eta>0$ the form as same as proposed in [2]. 
Theorem 2: If $\left\|W_{b} \Delta_{m}\right\|_{1} \leq 1 /\left\|\theta^{*}\right\|_{1}-\eta\left\|s W_{b}\right\|_{1}$

the system (29), (30) guarantee the following inequality, in other words, for $\forall t^{*} \geq 0$, all signals in the closed-loop plant are bounded and the tracking error $e=x(t)-x_{m}(t)$ meets:

$$
\left\|e_{t^{*}}\right\|_{\infty} \leq\left(\eta\left\|s W_{b}\right\|_{1}\left\|\left(\theta^{* T} X_{m}(t-\tau)-k_{0} r\right)_{t^{*}}\right\|_{\infty} /\left(1-\eta\left\|s W_{b}\right\|_{1}\left\|\theta^{*}\right\|_{1}-\left\|W_{b} \Delta_{m}\right\|_{1}\left\|\theta^{*}\right\|_{1}\right)+\left\|W_{b} \Delta_{m}\right\|_{1}\left\|\left(\theta^{* T} X_{m}(t-\tau)-k_{0} r\right)_{t^{*}}\right\|_{\infty}\right)
$$

where $W_{b}(s)=\left(s I-A_{m}\right)^{-1} b$ and $x_{m}(t)=\hat{x}(t)$ is the state of the reference model (10).

Proof: Applied the small-gain theorem and input-output stability results can get the proof.

It is unambiguous from Theorem 2 that the presence of the input filter. For example, the stability margin bound can be reduced and the bound for the standard of the tracking error increased where $\eta \neq 0$. Similar to the arguments presented in above and the bound of the uncertain parameter in (31) shows that removing the filter (using the corresponding MRAC). We clear show that the stability of the corresponding adaptive scheme $\left(\theta(t)=\theta^{*}\right.$ where $\left.\lim _{t \rightarrow \infty} \theta(t)=\theta^{*}\right)$ cannot be established where the inequality (31) dissatisfy. As a matter of fact that $\theta(t) \neq \theta^{*}$, further narrowed the stability margin bounds in (31) and requiring a more detailed analysis to establish them.

As it follows from the above analysis: the problem which has been amended in the robust adaptive control document did not amend with the $\mathrm{L}_{1}$-AC results, and the document is the stability and performance of adaptive control schemes for plant (29) designed for $\Delta_{m}(s)=0$ but analyzed for $\Delta_{m}(s) \neq 0$ with no knowledge of $\Delta_{m}(s)$. However, parameterized and expressed as $\theta(t)^{T} x(t-\tau)$ are the effect of multiplicative uncertainty, where $\theta(t)$ is unknown parameter and $x(t-\tau)$ is a known signal vector. This essentially converts the unmodeled dynamics which is a dynamic uncertainty into a parametric uncertainty; in such a case, all standard adaptive methods without any modification can be directly used. In some references, the knowledge of $\Delta_{m}(s)$ is based on the design filter $C(s)$.

As the illustrations of the analytical results we consider the plant $\dot{x}(t)=-x(t)+\theta^{*} x(t-\tau)+u(t), x(0)=1$, where $\theta^{*} \in[-3,3]$ is an unknown parameter. The reference model is $\dot{x}_{m}(t)=-x_{m}(t)+r(t)$ and $r(t)=5 \cos (0.5 t)$.

We assume that the input filter is of the form $C(s)=1 / \eta s+1$ for which the stability condition (12) is satisfied for any $\theta^{*} \in[-3,3]$, where $\eta<0.1$. We choose $\eta=0.05$, and assume $\theta^{*}=0$. Fig. 1 and Fig. 2 show the simulations generated using Matlab demonstrating the results that are consistent with what we have shown analytically. $\tau$ as small as possible, and we choose $\tau=2 * 10^{-4}$ sec and using a forth order Runge-Kutta method. Figure 1 shows that in the case of $\mathrm{L}_{1}$-AC, the tracking performance is lacked, and Figure 2 shows that MRAC (removal of the filter), the tracking performance is recovered.
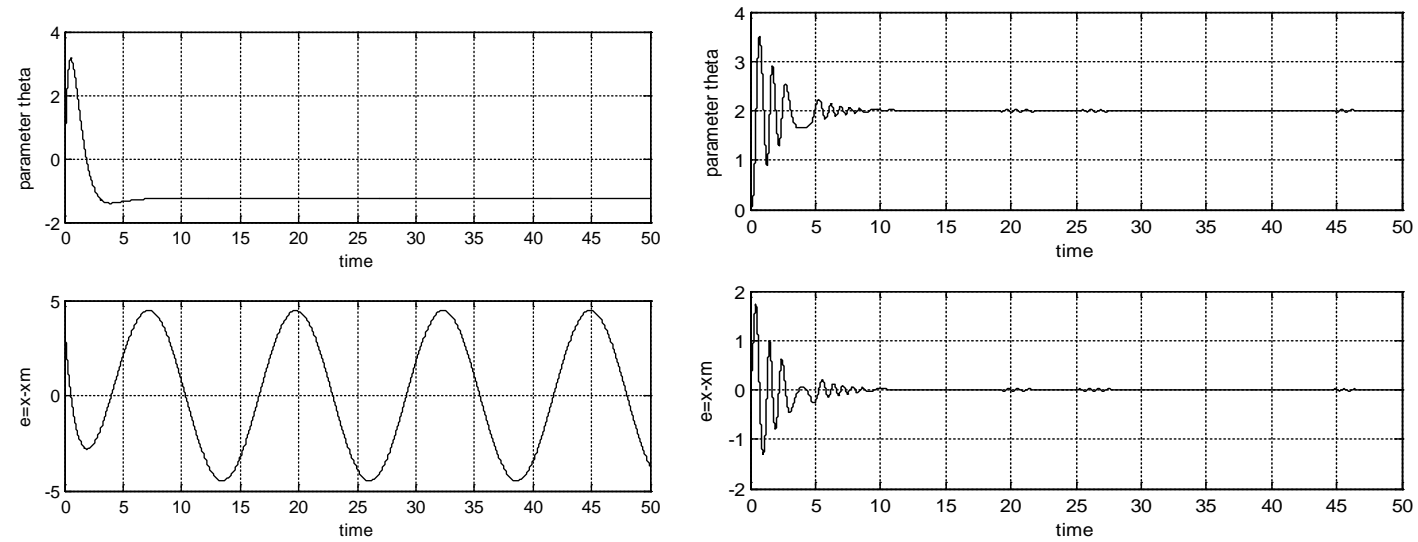

Fig.1: The tracking performance with filter Fig.2: The tracking performance without filter

In addition to all of the above, another negative effect of the use of high adaptive gains is the 'freezing' of the adaptive law as the adaptive gain becomes large, as discussed in [13]. 


\section{Conclusion}

In this paper, we analyze the stability and performance with state delay of an adaptive control design approach which was given the name $\mathrm{L}_{1}$-AC and led to a body of work involving different classes of plant. We have shown analytically that the $\mathrm{L}_{1}$-AC approach for a class of simple LTI plants with states accessible presented in [1], [12] offers no benefit in terms of performance, robustness or bounds that suggest useful trade-offs. On the contrary, the approach deteriorates the very properties of the MRAC that it is purportedly trying to improve.

\section{Acknowledgement}

In this paper, the research was sponsored by the National Nature Science Foundation(Project No. 61563031 and No.61263003) and the Natural Science Foundation of Gansu province under grant number 1208RJZA152

\section{References}

[1]C. Cao, N. Hovakimyan. Design and analysis of a novel L1 adaptive control architecture with guaranteed transient performance. IEEE Trans. on Automatic Control, 53(2) 586-591, 2008.

[2]C. Cao, N. Hovakimyan.Stability margins of L1 adaptive control architecture. IEEE Trans. on Automatic Control. 55(2)480-487, 2010.

[3]N. Hovakimyan and so on. L1 adaptive control for safety-critical systems. IEEE Control Systems Magazine. 31(5) 54-104, 2011.

[4]C. Cao, N. Hovakimyan. L1 adaptive output feedback controller for systems of unknown dimension. IEEE Trans. on Automatic Control. 53 (3) 815-821, 2008.

[5]A. Petersson and so on. Augmenting L1 adaptive control of piecewise constant type to a fighter aircraft. performance and robustness evaluation for rapid maneuvering. In Proc. of AIAA Guidance, Navigation, and Control Conference, 2012.

[6]K. van Heusden, G. Dumont.Analysis of L1 adaptive output feedback control; equivalent LTI controllers. In 16th IFAC Symposium on System Identification, 2012.

[7]P. A. Ioannou and so on. L1-adaptive control: Stability, robustness, and interpretations. IEEE Trans on Automatic Control. 59(11) 3075-3080, 2014.

[8]B. Egardt. Stability of adaptive controllers. New York: Springer Verlag, 1979.

[9]P. A. Ioannou, J. Sun. Robust adaptive control. Upper Saddle River, NJ: Prentice-Hall, 1996.

[10]K. S. Narendra, A. M. Annaswamy. Stable adaptive systems. Englewood Cliffs, NJ: Prentice Hall, 1989; Dover Publications, 2004.

[11]M. Krstic and so on. Nonlinear and adaptive control design. Wiley Interscience, 1995.

[12]K. Zhou and so on. Robust and optimal control. Prentice Hall, 1996.

[13]R. Ortega, E. Panteley. Comments on L1 adaptive control: Stabilization mechanism, existing conditions for stability and performance limitations. International Journal of Control. 87(3) 581-588, 2014. 